\title{
Simulation of radiation damage in rocks considered for safe storage of nuclear waste
}

\author{
V. I. Dubinko ${ }^{1}$, A. N. Dovbnya ${ }^{1}$, D. I. Vainshtein ${ }^{2}$ \\ \& H. W. den Hartog 2 \\ ${ }^{I}$ National Science Center Kharkov Institute of Physics and Technology, \\ 61108 Kharkov, Ukraine \\ ${ }^{2}$ Solid State Physics Laboratory, University of Groningen, Nijenborgh 4, \\ NL-9747 AG Groningen, The Netherlands
}

\begin{abstract}
Rock salt is one of the considered storage mediums for radioactive waste, and so the behavior of rock salt in the vicinity of the waste canisters is of great practical interest. The main contribution to the irradiation from a radioactive waste comes from gamma-radiation, which requires a comparison of its action with that of the electron irradiation, widely used for simulation of radiation damage in rock salt. Samples of synthetic crystals of $\mathrm{NaCl}$ have been electron and $\gamma$-irradiated and subsequently investigated by means of differential scanning calorimetry and atomic force microscopy. Our theoretical modeling of the irradiation damage has shown that the observed difference in colloid production under electron and gamma irradiation may be explained by the difference in the dose rates rather than the irradiation type. The data on radionuclide migration in the geological medium material of the disposal site are required for estimating the ecological safety of the radioactive waste disposal system in granite and tuff rocs. Penetration profiles of cerium ${ }^{139} \mathrm{Ce}$ in granite and tuff specimens (before and after $\gamma$-irradiation) were measured by means of nuclear physics methods.
\end{abstract}

Keywords: radioactive waste, electron and gamma-radiation, rock salt, tuff, granite, radionuclide migration.

\section{Introduction}

Rock salt is an attractive storage medium for nuclear high level waste (HLW) since geological rock salt deposits stay away from water for millions of years. 
However, in-laboratory electron irradiation has been shown to result in formation of radiolytic products, namely, nano-sized sodium colloids and chlorine bubbles, followed by formation of relatively large voids eventually bringing the halogen gas and metal to a back reaction inside the voids resulting in explosive fracture of samples with particular dopants Dubinko et al [1-3]. So the behavior of rock salt in the vicinity of HLW should be investigated very carefully.

One of the problems of imitation of actual conditions of irradiation is that the main contribution to the irradiation from a radioactive waste consists of gammaradiation, which requires a comparison of its action with that of the electron irradiation. To solve this problem, we have used linear electron accelerator ELIAS of the NSC KIPT to produce bremsstrahlung gamma-radiation spectrum similar to the real spectrum of HLW. Samples of synthetic crystals of $\mathrm{NaCl}$ (pure and doped by $\mathrm{KBF} 4, \mathrm{KCl}$, and $\mathrm{NaBr}$ ) have been $\gamma$-irradiated up to the dose ranging from $1.2 \times 10^{8}$ Gy to $7.8 \times 10^{8} \mathrm{~Gy}(12 \mathrm{Grad}$ to $78 \mathrm{Grad})$ and subsequently investigated by means of differential scanning calorimetry and atomic force microscopy. The results have been compared with those produced by electron irradiation of $\mathrm{NaCl}$ samples at the University of Groningen, and analyzed in the framework of a new theoretical model Dubinko et al $[4,5]$.

Another attractive storage medium for HLW is granite and tuff geological formations. Therefore, it appears rather important to clarify the mechanisms and the rate of radionuclide penetration into these materials. In cases of abnormal destruction of the metal container after storage time $\leq 500$ years, granite (or tuff) matrix, will be subjected mainly to $\gamma$-irradiation Dubinko et al [2].The structure of irradiated rock will be different from that of intact natural rock resulting in the difference in the actinide penetration rates depending on the magnitude of $\gamma$-irradiation dose. However, the influence of $\gamma$-irradiation on radionuclide migration in granite or tuff matrices has not been investigated to our knowledge.

In the second part of the present work the migration of $139 \mathrm{Ce}$ (the actinide simulator) in the natural granite and tuff matrices $\gamma$-irradiated to different absorbed doses is is to investigated.

\section{Simulation of radiation damage in rock salt}

\subsection{Electron irradiation}

The irradiation source consisted of a LINear electron ACcelerator (LINAC), driven by the high voltage produced by a cascade generator, which was enclosed by a steel tank and placed (for reasons of radiation safety) in a bunker. With this LINAC system we were able to irradiate a target area of $320 \mathrm{~cm}^{2}$ at dose rates between $10 \mathrm{Mrad} / \mathrm{hr}$ and $1.5 \mathrm{Grad} / \mathrm{hr}$ in the temperature interval $40-150^{\circ} \mathrm{C}$.

We have carried out four irradiation runs to different dose levels in the temperature range from 40 to $150^{\circ} \mathrm{C}$. As a result, a total number of 1335 samples (pure and alloyed by $\mathrm{KBF}_{4}, \mathrm{~K}, \mathrm{Br}, \mathrm{Ba}, \mathrm{Li}$ and $\mathrm{F}$ ) have been irradiated up to the total absorbed dose in the range from 20 to $1000 \mathrm{Grad}$ with the dose rate ranging from 240 to $1000 \mathrm{Mrad} /$ hour. 


\subsection{Gamma-irradiation}

For obtaining bremsstrahlung gamma-radiation spectrum that is similar to the one from a real radioactive waste we have used $3 \mathrm{MeV}$ electron beam produced by Van-de-Graaf electrostatic electron accelerator ELIAS of the model KS/3000. Electron beam was converted into a beam of gamma-quanta by a specially designed converter. The converter design was based on modeling results on $3 \mathrm{MeV}$ electron interaction with converter construction elements with account of radiation and radiation-chemical stability of converter construction elements. Thus $3 \mathrm{MeV}$ electron beam with current density $150 \mu \mathrm{A} / \mathrm{cm}^{2}$ was converted into gamma-quanta beam with intensity of about $10^{14} \gamma$-quanta $/ \mathrm{cm}^{2} \mathrm{sec}$.

A special thermostatic chamber for irradiation of rock-salt specimens has been designed and manufactured together with the converter. It allows irradiating four rolls of rock-salt tablet-shaped specimens with diameter of 5.5-6.0 mm by gamma-quanta simultaneously at a constant temperature. Each roll may contain up to 25 specimens of one material with thickness of $0.7 \mathrm{~mm}$.

The temperature was stabilized during irradiation at $100^{\circ} \mathrm{C}$ with accuracy of $1^{\circ} \mathrm{C}$. Along the specimen roll, the intensity of gamma-quanta decreased monotonously, which allowed obtaining specimens with different absorbed dose during one irradiation run.

In this study, 100 samples of synthetic crystals of $\mathrm{NaCl}$ (pure and alloyed by $\mathrm{KBF}_{4}, \mathrm{~K}$, and $\mathrm{Br}, 25$ samples of each kind) have been irradiated by $\gamma$-quanta at $100^{\circ} \mathrm{C}$ up to the total absorbed dose in the range from 12 to $78 \mathrm{Grad}$.

\subsection{Experimental methods of microstructure examination}

The irradiated samples have been examined by means of differential scanning calorimetry (DSC) and atomic force microscopy (AFM).

The AFM results were obtained in ultra high vacuum (UHV) using an UHV AFM/STM Omicron system in the non-contact mode. To avoid chemical reactions between the radiolytic $\mathrm{Na}$ colloids and oxygen or water, the irradiated samples were cleaved, prepared and kept under UHV conditions prior to and during the experiments. Several AFM images have been obtained from different areas for each sample. The radii of AFM tips, used in this investigation, have been estimated to be in the range between 20 and $30 \mathrm{~nm}$

DSC measurements are very suitable for the determination of the volume fraction of metallic Na produced inside the sample during the irradiation process. The latent heat of melting (LHM) of the Na colloids has been measured for each sample with a Perkin-Elmer DSC-7 system. The LHM is proportional to the volume fraction of the Na colloids so that the LHM equal to $1 \mathrm{~J} / \mathrm{g}$ corresponds approximately to the volume fraction of $2 \%$. The difference in heat flow between the irradiated sample and the reference sample (unirradiated $\mathrm{NaCl}$ ) was measured during heating from $50^{\circ} \mathrm{C}$ to $150^{\circ} \mathrm{C}$ with a heating rate of $10^{\circ} \mathrm{C} / \mathrm{min}$. The appearance of the LHM peaks in the 'DSC-spectrum' in this temperature interval is evidence for the presence of sodium colloids in the sample. 


\subsection{Comparison of results on electron and gamma irradiation}

The nano-particles of metallic sodium formed after electron and gamma irradiation of $\mathrm{NaCl}$ crystals, doped by $\mathrm{K}$ are visualized on the AFM images shown in Fig. 1. The precipitates shape and sizes are slightly distorted because of the convolution of the surface with the AFM tip with irregular-shaped apex. It can be seen that the sizes and the concentrations of sodium colloids formed after electron and gamma irradiation are comparable.

The dose dependence of the latent heat of melting (LHM) of the Na colloids in electron and gamma-irradiated $\mathrm{NaCl}$ crystals, doped by $\mathrm{K}$ is shown in Fig. 2.

It can be seen that the LHM (and consequently the volume fraction) of $\mathrm{Na}$ colloids produced by gamma irradiation at the maximum absorbed dose is lower than that produced by electron irradiation by a factor of two.
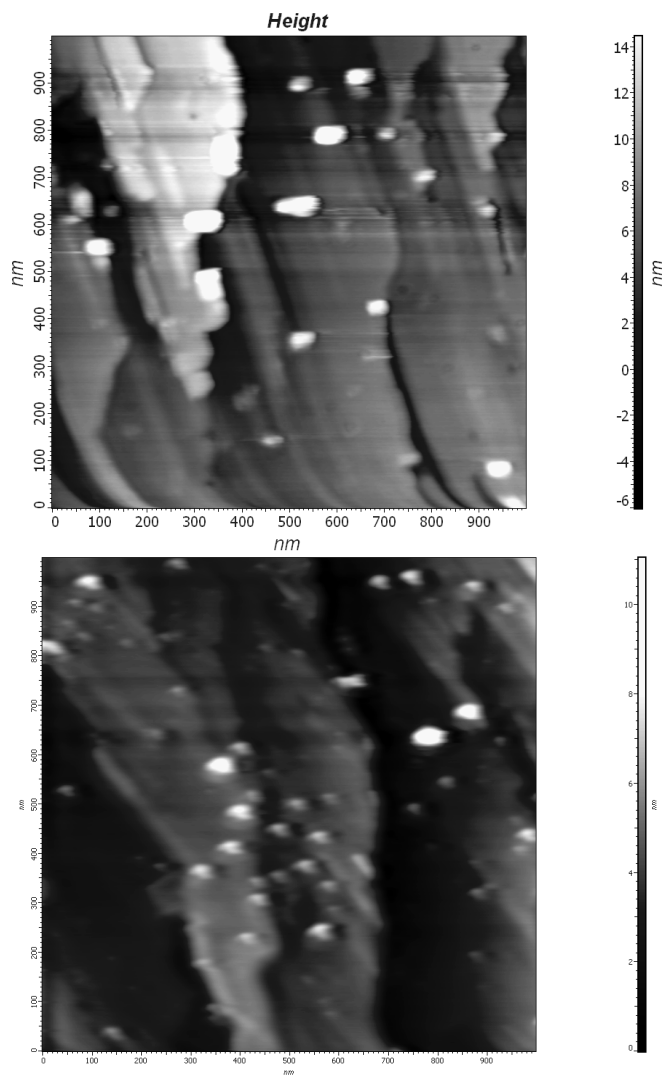

Figure 1: $\quad$ Typical AFM-images of $\mathrm{NaCl}$ crystals, doped by $\mathrm{K}$ and irradiated at $100^{\circ} \mathrm{C}$ up to $10 \mathrm{Grad}$ with electrons (the upper picture) and up to 11.4 Grad with gamma-quanta (the lower picture).

This difference can be explained in the framework of the model Dubinko et al $[4,5]$, as shown in Fig. 2, which compares the theoretical and experimental 
results. The model describes the evolution of sodium colloids, chlorine bubbles and voids under given irradiation conditions. The comparison between theoretical and experimental results shows that the observed difference in colloid production under electron and gamma irradiation may be explained by the difference in the dose rates rather than the irradiation type.

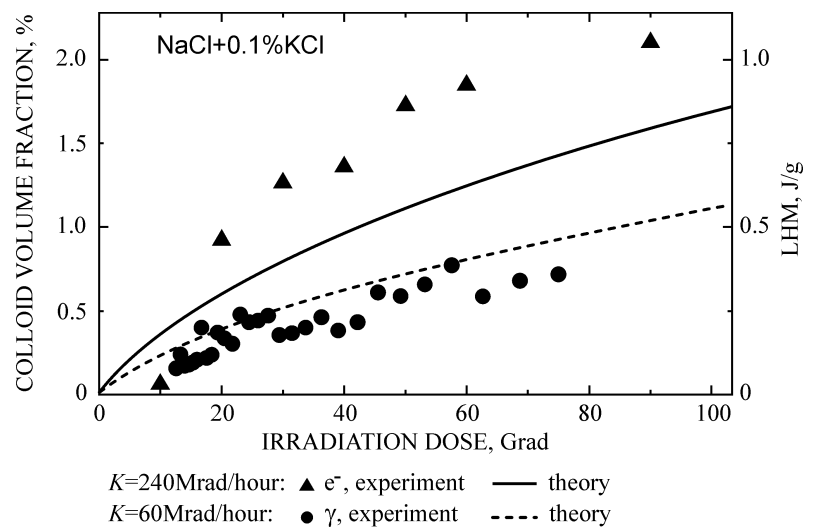

Figure 2: $\quad$ Dose dependence of the colloid volume fraction in $\mathrm{NaCl}+0.1 \% \mathrm{~K}$ at $100^{\circ} \mathrm{C}$ for different types and dose rates of irradiation: $\mathrm{K}=60$ $\mathrm{Mrad} / \mathrm{h}$ (gamma) and $240 \mathrm{Mrad} / \mathrm{h}$ (electron).

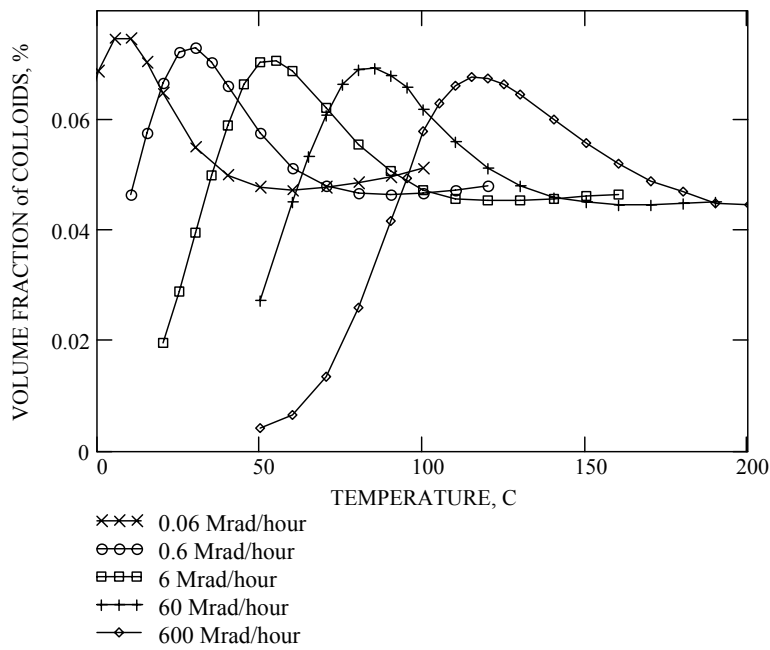

Figure 3: Temperature dependence of the colloid volume fraction in $\mathrm{NaCl}$ calculated at different dose rates for the total absorbed dose of 1 Grad.

The temperature dependence of the colloid volume fraction in $\mathrm{NaCl}$ calculated at different dose rates (Fig. 3) has a bell-shaped form, which shifts to 
higher temperatures with increasing dose rate. It means that in order to imitate irradiation conditions in HLW repository during the first hundred years (which corresponds to about $0.06 \mathrm{Mrad} /$ hour and to $50-100^{\circ} \mathrm{C}$ ) one should increase the temperature of in-laboratory irradiation $(60-600 \mathrm{Mrad} / \mathrm{hour})$ up to $150-200^{\circ} \mathrm{C}$. After a hundred years, the temperature around the HLW container is expected to saturate at about $40^{\circ} \mathrm{C}$, while the dose rate falls down to several $\mathrm{rad} / \mathrm{hour}$, which corresponds to approximately $300^{\circ} \mathrm{C}$ of in-laboratory irradiation.

\section{Investigation of actinide simulators migration in irradiated granite and tuff}

It is known that in the course of geological storage of high-level radioactive waste (HLW), such as spent nuclear fuel, etc., a failure of the metallic container (e.g. due to mechanical damage or corrosion) may give rise to the HLW contact with subsoil waters Forsyth et al [6]. Therefore, it appears rather important to clarify the mechanisms and the rate of radionuclide penetration into the surrounding matrix. In the present work, the migration of ${ }^{139} \mathrm{Ce}$ (the actinide simulator) in the natural granite and tuff matrices $\gamma$-irradiated to different absorbed doses is investigated.

\subsection{Starting materials and irradiation technique}

Cores of granite species from the Yantsev deposit of the Ukrainian crystalline stratum and tuff species from the Yucca Mountain deposit (Nevada, USA) were used as materials to study. The cores were cut into experimental specimens with dimensions of $10 \times 10 \times 30 \mathrm{~mm}$. The specimens were exposed to bremsstrahlung $\gamma$-irradiation produced at the electron linear accelerator.

As the $10 \mathrm{MeV}$ electron beam passes through a tantalum converter, it generates $\gamma$-quanta and neutrons produced in the reaction $\left(\gamma+{ }^{181} \mathrm{Ta} \rightarrow{ }^{180} \mathrm{Ta}+\mathrm{n}\right)$. The filter system (aluminum and paraffin) provides a substantial decrease in the electron and neutron constituents of the converted beam so that practically a pure $\gamma$-quantum beam with average energy of $2.0-2.3 \mathrm{MeV}$ is produced. The maximum absorbed dose rate in the present study was about $10^{4} \mathrm{~Gy} / \mathrm{h}$. The temperature of granite/tuff specimens during irradiation was about $50^{\circ} \mathrm{C}$.

\subsection{Analytical methods}

The migration ${ }^{139} \mathrm{Ce}$ deep into the rock matrix was investigated by several steps. First, the $\gamma$-tracer of ${ }^{139} \mathrm{Ce}$ was obtained using the following activation technique. Cerium oxide pellets $\left(\mathrm{CeO}_{2}\right)$ were irradiated with bremsstrahlung $\gamma$-quanta. In the course of irradiation, cerium isotopes ( $\gamma$-tracers) were produced in the reaction ${ }^{140} \mathrm{Ce}(\gamma, \mathrm{n}) \rightarrow{ }^{139} \mathrm{Ce}$ (the half-decay period, $\mathrm{T}_{1 / 2}=140$ days). Then the pellets were dissolved in a concentrated acid $\mathrm{H}_{2} \mathrm{SO}_{4}$ to make eventually the solution with $\mathrm{pH}=1.8$. Specimens under investigation (before and after irradiation) were placed in the solution and held for 180-550 hours at the room temperature. After that, the precise polishing was used to remove the material 
(layer by layer) from a free (uncoated with a protective compound) surface of the specimen. The thickness of the removed layers varied from 2 to $50 \mu$. The activity of the removed layer was measured with the use of a Ge(Li) $\gamma$ spectrometer. To estimate the migration of ${ }^{139} \mathrm{Ce}$, the spectra obtained in the measurements were used to plot the distribution of $\gamma$-tracer concentration in the depth of the specimen. The coefficients of cerium diffusion into the rock matrix were calculated using the obtained concentration profiles. Crystal-optical analysis was used to study the crystalline structure of granite and tuff matrices before and after irradiation.

\subsection{Results and discussion}

It is known that the penetration of a diffusant (in our case, cerium) into the other material is determined either by the grain bulk or grain boundary diffusion mechanisms Spasyonnykh [7]. These two mechanisms of diffusion (bulk and grain boundary) are called BD and GD, respectively, Geguzin [8].

Two characteristic regions, namely, 0 to $60 \mu$ and 60 to $900 \mu$, have been established in un-irradiated samples during the analysis of the curves showing the concentration distribution of cerium in the depth of the matrix (Fig. 1).

The first region is adequately described by BD. The average effective diffusion coefficients of cerium in initial granite and tuff specimens at a depth of $0-60 \mu$ were found to be $1.2 \cdot \times 10^{-15} \mathrm{~m}^{2} / \mathrm{s}$ and $2.9 \cdot \times 10^{-15} \mathrm{~m}^{2} / \mathrm{s}$, respectively.

In the 60-900 $\mu$ range, the cerium concentration distribution for all granite and tuff specimens is better described by GD. The average effective diffusion

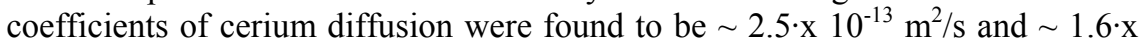
$10^{-10} \mathrm{~m}^{2} / \mathrm{s}$ for granite and tuff, respectively. Note that the grain boundary diffusion is much faster in tuff than in granite due to the higher porosity of tuff, which has been confirmed by the crystal-optical analysis.

The variations in the state of boundaries of natural materials under irradiation may substantially facilitate the penetration of cerium by the grain boundary diffusion mechanism. Besides, the structural defects formed under irradiation in the bulk (pores and cracks) may also facilitate the bulk diffusion. These expectations have been confirmed in our experiments, which have shown that after irradiation dose of $10^{7}$ Gy $(1 \mathrm{Grad})$, both granite and tuff specimens show substantial increase in the cerium penetration.

For irradiated granite specimens, the average effective diffusion coefficient was found to be $7.5 \times 10^{-14} \mathrm{~m}^{2} / \mathrm{s}$ (initial $1.2 \times 10^{-15} \mathrm{~m}^{2} / \mathrm{s}$ ) and $4.2 \cdot \times 10^{-8} \mathrm{~m}^{2} / \mathrm{s}$ (initial $2.5 \times 10^{-13} \mathrm{~m}^{2} / \mathrm{s}$ ) at penetration depths up to $60 \mu$ and $1600 \mu$, respectively.

For irradiated tuff specimens, the average effective diffusion coefficient was found to be $6.2 \cdot \times 10^{-14} \mathrm{~m}^{2} / \mathrm{s}$ (initial $2.9 \times 10^{-15} \mathrm{~m}^{2} / \mathrm{s}$ ) and $2.5 \times 10^{-8} \mathrm{~m}^{2} / \mathrm{s}$ (initial $1.6 \cdot \mathrm{x}^{-10} \mathrm{~m}^{2} / \mathrm{s}$ ) at penetration depths up to $60 \mu$ and $1800 \mu$, respectively.

Note that, in contrast to the initial state, the radiation-induced diffusion coefficients in granite are higher than those in tuff samples. It means that the effect of radiation on the microstructure is much stronger in granite than in tuff. 
This conclusion has been confirmed by further irradiation up the maximum absorbed dose of $3.0 \times 10^{8} \mathrm{~Gy}$.

At this dose, the coefficients of bulk and grain boundary diffusion in tuff were found to be $3.16 \times 10^{-14} \mathrm{~m}^{2} / \mathrm{s}$, and $1.26 \times 10^{-8} \mathrm{~m}^{2} / \mathrm{s}$, respectively, which were comparable with those obtained after irradiation dose of $10^{7} \mathrm{~Gy}$. So there is a tendency to saturation of diffusion coefficient growth with irradiation dose increasing beyond $10^{7} \mathrm{~Gy}$. The tuff specimens have retained their integrity, although the appearance of microcracks has been revealed by the crystal-optical analysis.

In contrast to that, under $\gamma$-irradiation to the maximum dose of $3.0 \times 10^{8} \mathrm{~Gy}$, structural changes in the granite specimens have caused their mechanical fracture into smaller pieces. The penetration of cerium through the fractured granite specimens has not been measured but evidently it should be much higher than the penetration through the whole tuff matrix.

\subsection{Estimation of the actinide penetration in tuff surrounding HLW}

In our experiments, cerium is the analog of actinides, namely, plutonium. We have used the radiation-induced Ce diffusion coefficients obtained in our experiments, $\mathrm{D}=1.26 \times 10^{-8} \mathrm{~m}^{2} / \mathrm{s}$, in order to estimate the penetration depth of plutonium isotopes in natural tuff massifs. The time dependence of the radionuclide penetration depth is given by $\lambda_{\text {diff }}(t)=2 \cdot(D t)^{1 / 2}[8]$. The characteristic time, during which the actinides preserve the radioactivity, is usually taken as $10 \cdot T_{1 / 2}$, where $T_{1 / 2}$ is the half-decay period. The estimated values of $\lambda_{\text {diff }}\left(10 \cdot \mathrm{T}_{1 / 2}\right)$ for different $\mathrm{Pu}$ isotopes are different due to the difference in their $\mathrm{T}_{1 / 2}$, which are as follows (in years): $\left.14.4\left({ }^{241} \mathrm{Pu}\right), 87.7{ }^{238} \mathrm{Pu}\right), 6537\left({ }^{240} \mathrm{Pu}\right)$ and $24000\left({ }^{239} \mathrm{Pu}\right)$. Accordingly, $\lambda_{\text {diff }}\left(10 \cdot \mathrm{T}_{1 / 2}\right)$ is given by (in meters) 15.13 $\left({ }^{241} \mathrm{Pu}\right), 37.34\left({ }^{238} \mathrm{Pu}\right), 322.34\left({ }^{240} \mathrm{Pu}\right)$ and $617.62\left({ }^{239} \mathrm{Pu}\right)$.

Therefore, the recommended distance of the HLW storage place from the ground surface in tuff is about or larger than $600 \mathrm{~m}$.

\section{Conclusions}

Colloid production under electron and gamma irradiation of rock salt does not depend on the irradiation type.

Irradiation of granite and tuff specimens to doses of $10^{7}$ Gy has increased the diffusion coefficients of actinides, which became larger in granite than in tuff samples. It means that the effect of irradiation on the microstructure is much stronger in granite than in tuff.

The diffusion coefficient growth in tuff with irradiation dose shows a tendency towards saturation beyond $10^{7} \mathrm{~Gy}$.

The tuff specimens have retained their integrity under $\gamma$-irradiation to the maximum dose of $3.0 \times 10^{8} \mathrm{~Gy}$, although the appearance of microcracks has been revealed. In contrast to that, under $\gamma$-irradiation to the maximum dose of $3.0 \times 10^{8} \mathrm{~Gy}$, structural changes in the granite specimens have caused their mechanical fracture. 
The recommended distance of the HLW storage place from the ground surface in tuff is about or larger than $600 \mathrm{~m}$.

\section{References}

[1] Dubinko V. I., Turkin A. A., Vainshtein D. I., H. W. den Hartog, Theory of the late stage of radiolysis of alkali halides. J. Nucl. Mater. 227, pp. 184 198, 2000.

[2] Dubinko V. I., Turkin A. A., Vainshtein D. I., H. W. den Hartog, Diffusion plus explosion mechanism of void-crack transition in irradiated $\mathrm{NaCl}$. Rad. Eff. and Defects in Solids, 156, pp. 27-31, 2001.

[3] Dubinko V. I., Turkin A. A., Vainshtein D. I., H. W. den Hartog, Critical parameters controlling mechanical stability of $\mathrm{NaCl}$ under irradiation. Rad. Eff. and Defects in Solids, 156, pp. 51-57, 2001.

[4] Dubinko V. I., Turkin A. A., Abyzov A. S., Sugonyako A.V., Vainshtein D. I., H. W. den Hartog, Nucleation and growth of sodium colloids in $\mathrm{NaCl}$ under irradiation: theory and experiment. Phys.Stat.Sol. (c), 2(1), pp. 438443, 2005.

[5] Dubinko V. I., Vainshtein D. I., H. W. den Hartog, Mechanism of void growth in irradiated $\mathrm{NaCl}$ based on exiton-induced formation of divacancies at dislocations. Nuclear Inst. and Methods in Physics Research, B228, pp. 304-308, 2005.

[6] Forsyth R.S., Werme L.O. Spent fuel corrosion and dissolution. J. Nucl. Mater. v. 190, pp. 3, 1992.

[7] Spasyonnykh M.Yu. The influence of water-rock interaction conditions on radionuclide migration in water-saturated rocks (in Russian). Geokhimiya, 2, pp. 218-226, 1997.

[8] Geguzin Ya.E. Diffusion zone (in Russian).ed. Nauka Moscow, pp. 40-41, 1979. 